\title{
A Novel Grip-Type Microsurgical Needle Holder: Introduction and Electromyographic Evaluation
}

\author{
Yoshichika Yasunaga, MD, PhD ${ }^{1,2}$ Daisuke Yanagisawa, MD ${ }^{2}$ Erika Ohata, MD \\ Shunsuke Yuzuriha, MD, PhD ${ }^{2}$ Shoji Kondoh, MD, $\mathrm{PhD}^{1}$ Kiyoshi Matsuo, MD, PhD 2,4
}

1 Division of Plastic and Aesthetic Surgery, Ina Central Hospital, Ina, Japan
2 Department of Plastic and Reconstructive Surgery, Shinshu
University Graduate School of Medicine, Matsumoto, Japan
${ }^{3}$ Division of Plastic Surgery, Toyama Nishi General Hospital, Toyama, Japan
${ }^{4}$ Matsuo Plastic and Oculoplastic Surgery Clinic, Hamamatsu, Japan

Address for correspondence Yoshichika Yasunaga, MD, PhD, Division of Plastic and Aesthetic Surgery, Ina Central Hospital, 1313-1 Koshirokubo, Ina, Nagano 396-8555, Japan (e-mail: yysng@shinshu-u.ac.jp).

J Reconstr Microsurg Open 2020;5:e50-e56.

\begin{abstract}
Keywords

- needle holder

- microsurgery

- electromyography
\end{abstract}

Employed in general surgery with a grasped-in-hand style, the Mathieu needle holder uses forearm and wrist movements to advance the needle. In contrast, pen-type microsurgical needle holders, such as the Castroviejo, ${ }^{1}$ the Barraquer, Jacobson, and bayonet types, primarily utilize finger twisting between the thumb and index or middle fingers. Surgeons manipulate the holder much like handling a pen or chopsticks with the intrinsic muscles of the hand, as well as the forearm muscles. ${ }^{2-4}$ We previously demonstrated by surface electromyography (sEMG) that an enlarged pentype needle holder, which we developed for such delicate operations as cleft lip and palate repairs and blepharoplasty, reduced forearm muscle movement during skin suturing as compared with a conventional Webster needle holder. ${ }^{5}$ received

June 16,2020

accepted

July 9,2020
DOI https://doi.org/

10.1055/s-0040-1715582. ISSN $2377-0813$.
Copyright $\odot 2020$ by Thieme Medical

Publishers, Inc., 333 Seventh Avenue, New York, NY 10001, USA. Tel: +1(212) 760-0888.
License terms

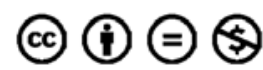


Based on that result, we hypothesized that a needle holder which required only finger twisting for needle advancement would be better suited for microsurgery owing to decreased forearm muscle movement and more stable microsurgical suturing. To verify this concept, we have newly developed a gun-shaped microsurgical needle holder that is held by its grip, thus coined as "grip-type." The purpose of this study was to objectively assess whether the grip-type needle holder reduced forearm muscle movement during microsurgical suturing in a laboratory setting as compared with a conventional pen-type needle holder in terms of sEMG, an established indicator of muscle movement. ${ }^{6}$

\section{Materials and Methods}

\section{Instrument Description}

The newly developed microsurgical needle holder (grip-type needle holder type-MY; Nomura Medical Device Corp., Chino, Japan) consists of a 7.6-cm grip, an 11.5-cm rotating shaft with needle-grasping straight jaws on its tip, and a trigger to release the needle (-Fig. 1A, B). Surgeons hold the grip firmly with the ring and little fingers and place the hand on the patient for stabilization. The length of the shaft is decided as the distance between the surgeon's hand and the needle in a manner comparable to that of the widely used $13-\mathrm{cm}$ pen-type microsurgical needle holder. To advance the semicircular needle, surgeons have only to twist the thumb and index finger on the shaft with the intrinsic muscles of hand for shaft rotation, with no need for movement of the wrist or forearm (-Fig. 1C; - Video 1). The shaft can rotate 180-degree symmetrically from 90-degree backward to 90-degree forward. The needle is held between the holder's jaws by a pair of leaf springs without the need for a ratchet mechanism, which are smoothly released by pulling on the trigger with the middle finger. Regardless of whether the
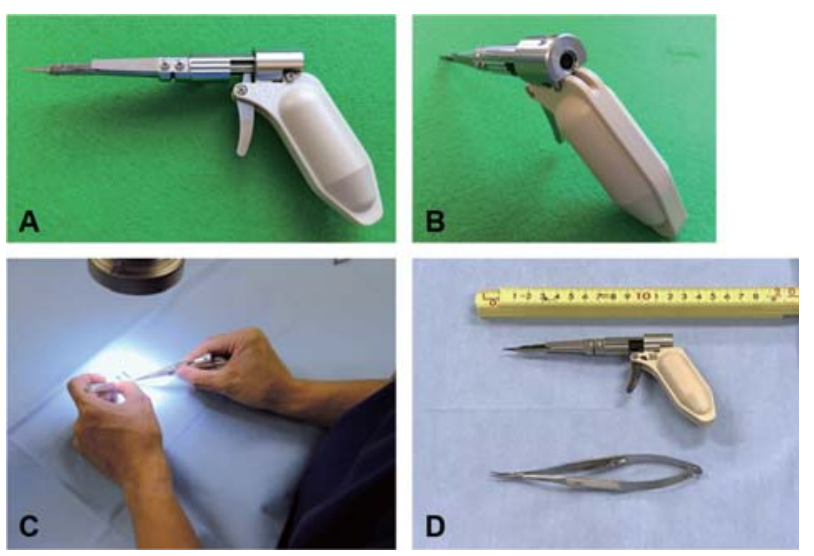

Fig. 1 Newly developed grip-type microsurgical needle holder. (A, B) The grip-type needle holder consists of a 7.6-cm grip, an 11.5-cm rotating shaft with needle-grasping straight jaws on its tip, and a trigger to release the needle. (C) Surgeons hold the grip firmly with the ring and little fingers and twist the thumb and index finger on the shaft to advance the needle. (D) Comparison of the grip-type holder with the conventional pen-type microsurgical needle holder employed in this study. surgeon is right handed or left handed, both antegrade and retrograde needle advancements are equally possible.

\section{Video 1}

Movement of the surgeon's hand and microsuture during needle advancement. Surgeons advance the needle by finger twisting only with the grip-type holder, but mainly by forearm movement with the pen-type holder. Online content including video sequences viewable at: https://www.thieme-connect.com/ products/ejournals/html/10.1055/s-0040-1715582.

\section{Suturing Materials}

The grip-type microsurgical needle holder and a conventional 13-cm curved tip, round-handled, pen-type microsurgical needle holder with a locking mechanism (N-2377-R; Keisei Medical Industrial Co., Ltd., Tokyo, Japan; - Fig. 1D) were employed in this study. We used 10-0 microsuture and a semicircular needle with a length of $4 \mathrm{~mm}$ and a curvature radius of 135 degrees, both of which were the same as those used in microvascular anastomosis such as free flap transfer and finger replantation. The training vessel graft used for anastomosis (Hybridgraft H200-12C; ACP Japan Co., Ltd., Tokyo, Japan) was of $2.00 \mathrm{~mm}$ caliber.

\section{Tasks}

We designed a microvascular anastomosis task in a laboratory setting to include a needle penetrating one end of two aligned training vessel grafts and being removed out of the other end under microscopic visualization (-Fig. 2).

\section{Task 1: Comparison of Eight Subjects for Horizontal} Needle Advancement

Eight right-handed plastic surgeons (subjects 1-8) were recruited for task 1 . Both grafts were placed horizontally and each subject advanced the needle from right to left (-Fig. 2A).

Task 2: Comparisons among Five Suturing Directions by One Subject

Task 2 was performed by a right-handed plastic surgeon with 9 years of microsurgery experience (Subject 1 ) and included five different suturing directions: (1) both grafts were placed horizontally and the subject advanced the needle from right to left (0 degrees; - Fig. 2A), (2) the right end was placed at 30-degree upward and the subject advanced the needle from right to left (+30 degrees; - Fig. 2B), (3) the right end was placed at 30-degree downward and the subject advanced the needle from right to left (-30 degrees; - Fig. 2C), (4) both grafts were placed longitudinally and the subject advanced the needle toward himself (90-degree antegrade; - Fig. 2D), and (5) both grafts were placed longitudinally and the subject advanced the needle away from himself (90-degree retrograde; - Fig. $\mathbf{2 E}$ ). 

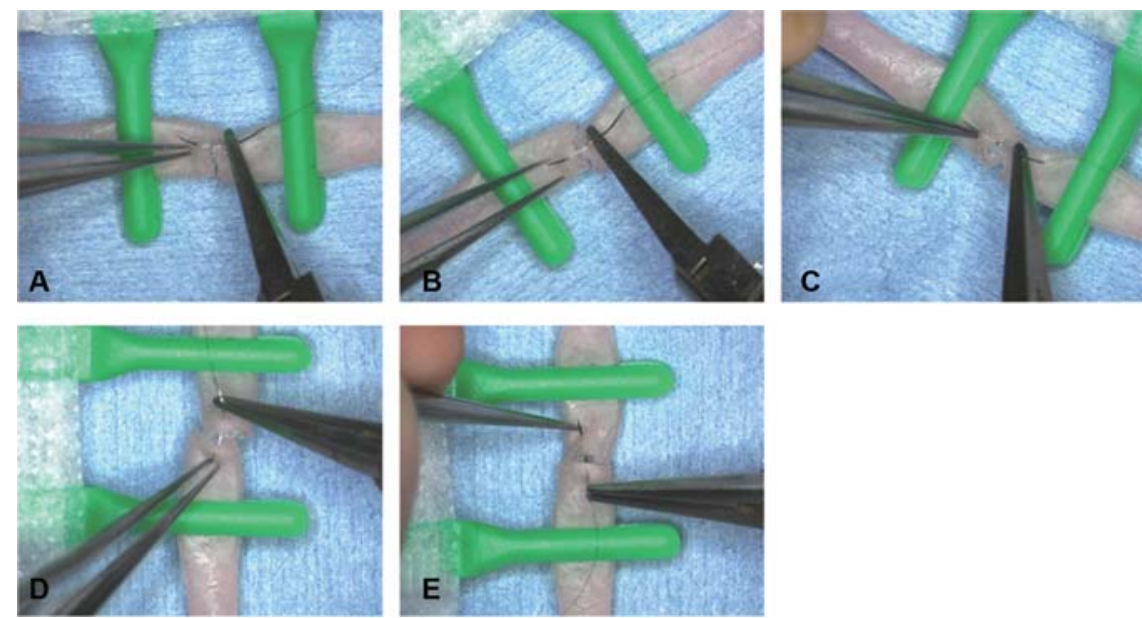

Fig. 2 Suture directions in tasks 1 and 2. (A) 0 degrees: both grafts were placed horizontally and the subject advanced the needle from right to left. (B) +30 degrees: the right end was placed 30 degrees upward and the subject advanced the needle from right to left. (C) -30 degrees: the right end was placed 30 degrees downwards and the subject advanced the needle from right to left. (D) 90-degree antegrade: both grafts were placed longitudinally and the subject advanced the needle toward himself. (E) 90-degree retrograde: both grafts were placed longitudinally and the subject advanced the needle away from himself.

\section{Surface Electromyography}

The applicability and measurement details of sEMG in this study have been described in our previous article. ${ }^{5}$ Briefly, extensor carpi ulnaris muscle (ECU) and flexor carpi ulnaris muscle (FCU) activity were assessed as representative muscles of dorsiflexion and palmar flexion of the wrist, respectively. ${ }^{7}$ We recorded SEMG using two compact electrode telemeters (ZB-150H; Nihon Kohden, Tokyo, Japan;
-Fig. 3A) and a host computer for real-time display and data storage (WEB-1000; Nihon Kohden, Tokyo, Japan). The telemeters were placed on the skin just above the centers of the ECU and FCU (-Fig. 3B, C). The sEMG signals taken during needle advancement (i.e., from penetrating one graft to being removed out of the other) were isolated ( - Fig. 3D), and root mean square (RMS) values of the extracted SEMG signals were calculated.
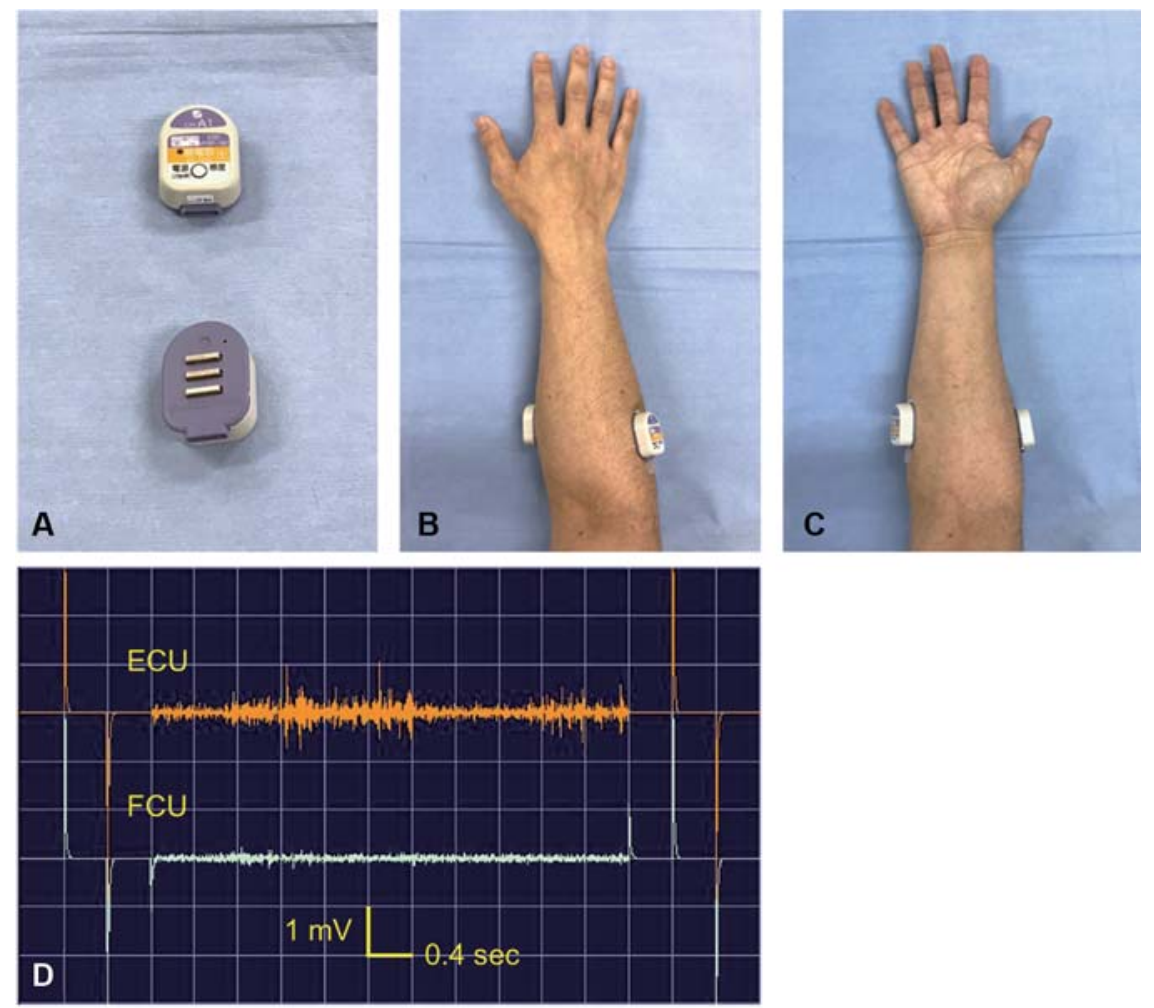

Fig. 3 Surface electromyography (sEMG). (A) The compact electrode telemeters. (B, C) The telemeters were placed on the skin just above the centers of the right ECU and FCU. (D) Signals of sEMG from the time of suture penetration of one graft to removal out of the other graft. ECU, extensor carpi ulnaris muscle; FCU, flexor carpi ulnaris muscle. 
The RMS values of the ECU and FCU obtained from 10 consecutive trials with each needle holder were averaged by subject or suturing direction. The summed ECU and FCU RMS values were then used as indicators of forearm muscle movement during needle advancement.

\section{Statistical Analysis}

All values are expressed as the mean \pm standard deviation. Differences between RMS values for the pen-type and griptype needle holders were analyzed using Student's $t$-test or the Wilcoxon's signed-rank test, as appropriate. Intergroup differences based on the subjects' years of microsurgery experience (less than 3 years and 3 years or more) were analyzed by Fisher's exact probability test. One-way analysis of variance (ANOVA) was adopted to compare means between the five suturing directions. When ANOVA produced a significant result, post hoc multiple comparisons were performed with Tukey's honest significant difference test.

A $p$-value of $<0.05$ was considered statistically significant. All statistical analyses were conducted using SPSS PASW Statistics version 26.0 software (IBM Inc., Armonk, NY).

\section{Results}

\section{Surface Electromyography Measurements of Eight Subjects in Horizontal Needle Advancement}

The characteristics and RMS values of each subject are summarized in - Table 1 . The mean duration of microsurgery experience was $4.0 \pm 3.2$ years. The mean summed ECU and FCU RMS values for the grip-type holder were significantly smaller than those for the pen-type holder $(p<0.05$; - Fig. 4). While the summed RMS values were lower for the grip-type holder in four subjects (subjects 1, 3, 4, and 5), no subject displayed smaller values for the pen-type holder. Two subjects (subjects 3 and 8 ) exhibited very high RMS values due to well-developed musculature and thin subcutaneous fat. The summed RMS values were lower for the grip-type holder in four of the five subjects with 3 or more years of microsurgery experience, while none of the three subjects with less than 3 years of experience showed a

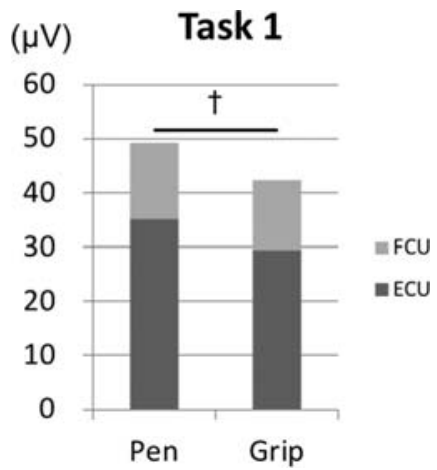

Fig. 4 Task 1: RMS values of each needle holder by eight subjects. The summed ECU and FCU RMS values for the grip-type holder were smaller than those for the pen-type holder. ECU, extensor carpi ulnaris muscle; FCU, flexor carpi ulnaris muscle, RMS, root mean square; ${ }^{\dagger} p<0.05$ by the Wilcoxon's signed-rank test.

remarkable difference in summed RMS values between the holder types. The difference in summed RMS values by the subjects' years of experience (i.e., less than 3 years or 3 or more years) was not significant $(p=0.07)$.

\section{Surface Electromyography Measurements of One Subject in Five Suturing Directions}

The mean RMS values for each suturing direction by subject 1 are listed in - Table 2 . The summed ECU and FCU RMS values for each suturing direction were smaller for the grip-type holder (all $p<0.05$; - Fig. 5), with the largest difference observed at -30 degrees ( - Fig. $\mathbf{5 C}$ ).

The results of one-way ANOVA and multiple comparisons of RMS values for the needle holders are shown in - Table 3 and -Fig. 6. All one-way ANOVAs of RMS values were significant apart from that of the grip-type FCU, indicating that it was consistent among the five suturing directions. In multiple comparisons, the summed RMS value at -30 degrees was greater than that at 0 degrees for the pen-type holder, which was not seen for the grip-type holder (-Fig. 6). Additionally, whereas the FCU RMS value was greater than the ECU RMS value at 90-degree antegrade for the pen-type holder, the opposite

Table 1 RMS values $(\mu \mathrm{V})$ of eight subjects during horizontal needle advancement

\begin{tabular}{|l|l|l|l|l|l|l|l|}
\hline \multicolumn{3}{|c|}{ Subject } & \multicolumn{3}{c|}{ Pen type } & \multicolumn{3}{c|}{ Grip type } \\
\hline No. & Experience $(y)$ & ECU & FCU & Sum & ECU & FCU & Sum \\
\hline 1 & 9 & $12.5 \pm 1.4$ & $12.6 \pm 2.2$ & $25.1 \pm 2.7$ & $13.7 \pm 1.6$ & $7.1 \pm 0.6^{\mathrm{a}}$ & $20.8 \pm 1.9^{\mathrm{a}}$ \\
\hline 2 & 8 & $32.9 \pm 3.4$ & $14.8 \pm 5.1$ & $47.7 \pm 5.0$ & $29.3 \pm 5.4$ & $15.7 \pm 7.4$ & $45.0 \pm 12.1$ \\
\hline 3 & 5 & $77.1 \pm 10.8$ & $10.0 \pm 0.8$ & $87.1 \pm 10.5$ & $55.0 \pm 13.5^{\mathrm{a}}$ & $11.0 \pm 1.6$ & $66.0 \pm 14.0^{\mathrm{a}}$ \\
\hline 4 & 4 & $23.7 \pm 3.6$ & $16.9 \pm 2.3$ & $40.6 \pm 4.6$ & $16.6 \pm 2.3^{\mathrm{a}}$ & $15.1 \pm 2.2$ & $31.7 \pm 2.4^{\mathrm{a}}$ \\
\hline 5 & 3 & $13.6 \pm 1.2$ & $9.8 \pm 0.8$ & $23.4 \pm 1.2$ & $9.7 \pm 1.8^{\mathrm{a}}$ & $10.1 \pm 1.1$ & $19.8 \pm 2.3^{\mathrm{a}}$ \\
\hline 6 & 1 & $18.6 \pm 2.6$ & $17.2 \pm 2.0$ & $35.8 \pm 3.9$ & $14.6 \pm 1.4^{\mathrm{a}}$ & $18.5 \pm 1.6$ & $33.1 \pm 2.6$ \\
\hline 7 & 1 & $12.4 \pm 2.7$ & $8.1 \pm 0.6$ & $20.5 \pm 3.1$ & $14.2 \pm 1.9$ & $7.7 \pm 0.7$ & $21.9 \pm 2.6$ \\
\hline 8 & 1 & $90.2 \pm 14.6$ & $23.6 \pm 6.6$ & $113.8 \pm 20.8$ & $82.9 \pm 11.6$ & $18.2 \pm 3.9^{\mathrm{a}}$ & $101.1 \pm 13.5$ \\
\hline Mean & $4.0 \pm 3.2$ & $35.1 \pm 30.9$ & $14.1 \pm 5.1$ & $49.3 \pm 33.7$ & $29.5 \pm 26.1^{\mathrm{b}}$ & $12.9 \pm 4.5$ & $42.4 \pm 28.3^{\mathrm{b}}$ \\
\hline
\end{tabular}

Abbreviations: ECU, extensor carpi ulnaris muscle; FCU, flexor carpi ulnaris muscle; RMS, root mean square.

${ }^{a} p<0.05$ by Student's $t$-test versus the pen-type holder.

b $p<0.05$ by the Wilcoxon's signed-rank test versus the pen-type holder. 
Table 2 RMS values $(\mu \mathrm{V})$ of one subject in five suturing directions

\begin{tabular}{|l|l|l|l|l|l|l|}
\hline \multirow{2}{*}{ Suture direction } & \multicolumn{3}{|c|}{ Pen type } & \multicolumn{3}{c|}{ Grip type } \\
\cline { 2 - 7 } & ECU & FCU & Sum & ECU & FCU & Sum \\
\hline 0 degrees & $27.7 \pm 2.2$ & $10.8 \pm 1.8$ & $38.5 \pm 3.2$ & $18.2 \pm 5.1^{\mathrm{a}}$ & $7.6 \pm 1.9^{\mathrm{a}}$ & $25.8 \pm 5.4^{\mathrm{a}}$ \\
\hline+30 degrees & $25.4 \pm 1.2$ & $11.7 \pm 3.3$ & $37.1 \pm 3.8$ & $12.7 \pm 1.6^{\mathrm{a}}$ & $6.9 \pm 0.3^{\mathrm{a}}$ & $19.6 \pm 1.8^{\mathrm{a}}$ \\
\hline -30 degrees & $37.4 \pm 2.3$ & $12.8 \pm 4.3$ & $50.2 \pm 4.7$ & $18.1 \pm 3.1^{\mathrm{a}}$ & $7.1 \pm 0.3^{\mathrm{a}}$ & $25.2 \pm 3.4^{\mathrm{a}}$ \\
\hline 90-degree antegrade & $11.4 \pm 1.1$ & $20.9 \pm 1.8$ & $32.3 \pm 2.4$ & $8.3 \pm 0.5^{\mathrm{a}}$ & $7.1 \pm 0.9^{\mathrm{a}}$ & $15.4 \pm 1.1^{\mathrm{a}}$ \\
\hline 90-degree retrograde & $15.6 \pm 2.0$ & $7.7 \pm 1.6$ & $23.3 \pm 2.3$ & $9.0 \pm 1.4^{\mathrm{a}}$ & $7.0 \pm 0.5$ & $16.0 \pm 1.5^{\mathrm{a}}$ \\
\hline
\end{tabular}

Abbreviations: ECU, extensor carpi ulnaris muscle; FCU, flexor carpi ulnaris muscle; RMS, root mean square.

${ }^{a} p<0.05$ by Student's $t$-test versus the pen-type holder.
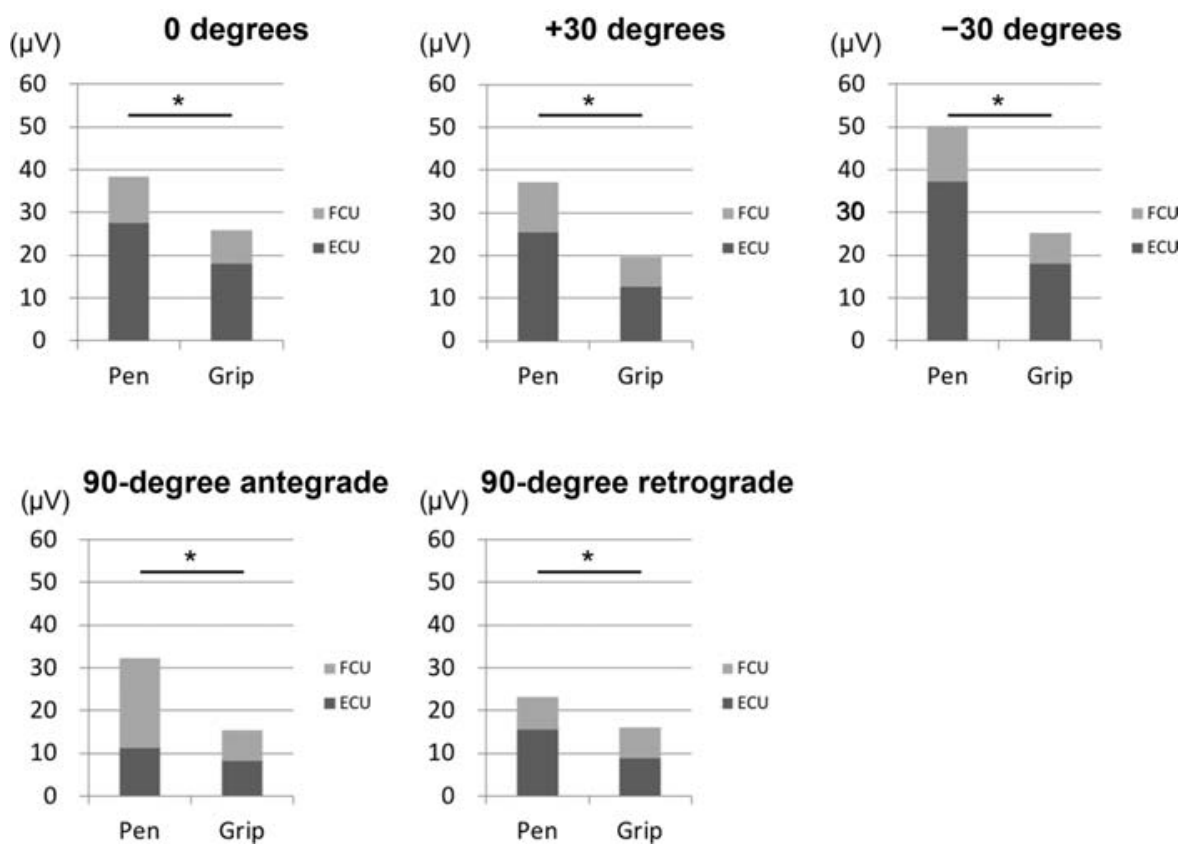

Fig. 5 Task 2: RMS values of each needle holder for each suturing direction by subject 1 . The summed ECU and FCU RMS values for each suturing direction were smaller for the grip-type holder. The summed RMS value for the pen-type holder decreased the most at -30 degrees. ECU, extensor carpi ulnaris muscle; FCU, flexor carpi ulnaris muscle, RMS, root mean square; ${ }^{*} p<0.05$ by Student's $t$-test.
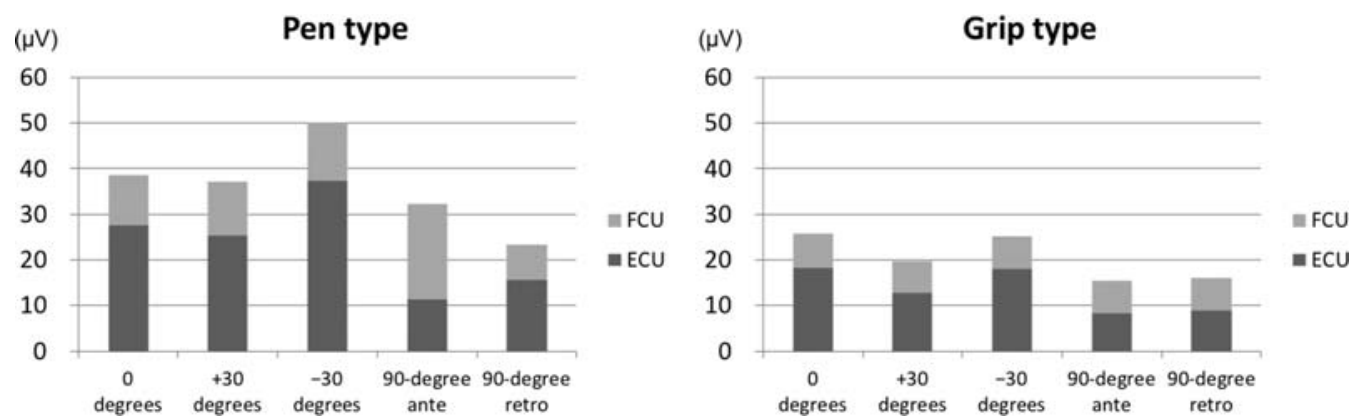

Fig. 6 Task 2: comparisons of RMS values between five suturing directions for each needle holder. For both needle holders, the summed ECU and FCU RMS values were smaller in the longitudinal directions (90-degree antegrade and 90-degree retrograde) than in the horizontal directions (0, +30 , and -30 degrees) except for the comparison of +30 - and 90 -degree retrograde for the grip-type holder. The summed RMS values were the smallest in the longitudinal directions (90-degree antegrade and 90-degree retrograde) for the grip-type holder. To avoid complicating $\mathbf{- F i g . 6}$, the significant differences in multiple comparisons of the summed ECU and FCU RMS values are not indicated as in - Figs. $\mathbf{4}$ and $\mathbf{5}$. The precise statistical comparisons are summarized in - Table 3. ante, antegrade; ECU, extensor carpi ulnaris muscle; FCU, flexor carpi ulnaris muscle; retro, retrograde; RMS, root mean square. 
relationship was observed at 90-degree retrograde ( - Fig. 6A). This phenomenon was absent for the grip-type holder (-Fig. 6B), which indicated that it did not involve forearm muscle movement in addition to finger twisting. For both needle holders, the summed RMS values were smaller in the longitudinal directions (90-degree antegrade and 90-degree retrograde) than in the horizontal directions $(0,+30$, and -30 degrees) except for the comparison of +30 - and 90 -degree retrograde for the grip-type holder. The summed RMS values were the smallest in the longitudinal directions (90-degree antegrade and 90-degree retrograde) for the grip-type holder (-Fig. 6B).

\section{Discussion}

In this study, objective sEMG measurements of eight subjects performing microsuturing tasks confirmed that the newly developed grip-type microsurgical needle holder could reduce the forearm muscle movement required for needle advancement as compared with a conventional pen-type instrument (-Fig. 4). Accordingly, the grip-type holder may be more ideally suited for delicate microsurgical suturing since the decreased forearm movement can mitigate the risk of coarse motion and hand shaking. Comparisons between five suturing directions demonstrated that the grip-type holder was particularly advantageous in the left-upward direction (-30 degrees) for right-handed surgeons, as well as in the longitudinal directions (90-degree antegrade and 90-degree retrograde). The left-upward direction is generally considered the most difficult for right-handed surgeons, as evidenced by the sEMG findings for the pen-type holder in this study (-Fig. 6A).

The core concept in the development of the grip-type needle holder was to minimize forearm muscle movement during needle advancement by fully utilizing finger twisting. The pen- type holder necessitates wrist palmar flexion with forearm supination for antegrade needle advancement and wrist dorsiflexion with forearm pronation for retrograde advancement in addition to finger twisting. In contrast, the grip-type holder requires only finger twisting involving thumb adduction and index finger flexion for antegrade advancement and thumb abduction and index finger extension for retrograde advancement without concerted forearm or wrist movement (- Fig. 1C, - Video 1). Most of these finger twisting movements are produced by the intrinsic muscles of the hand, such as the adductor pollicis, abductor pollicis brevis, dorsal and palmar interossei, and lumbricals. Among them, the lumbrical muscles have the greatest number of muscle spindles among upper limb muscles; therefore, although their biomechanical contribution is secondary due to their small size, they are considered to factor prominently in proprioceptive monitoring for precision movements of the fingers. ${ }^{8,9}$ We considered that the efficient use of these intrinsic muscles enabled the grip-type holder to spare forearm muscle movement by contributing principally to stable microsurgical suturing. Releasing the needle with the grip-type needle holder requires flexion of the middle finger to pull on the trigger with the extrinsic forearm flexors of the flexor digitorum superficialis and profundus. Although this needle-releasing motion was contained in the study tasks, the summed RMS values of the grip-type holder appeared not to be increased by this motion. Moreover, judging from the fact that the grip-type FCU values of the five suturing directions showed no intragroup differences (-Table 3; - Fig. 6B), we presumed that those five values $(6.9-7.6 \mu \mathrm{V})$ represented the baseline drift of SEMG and could be considered virtually zero. Likewise, the grip-type ECU values at 90-degree antegrade and 90-degree retrograde ( 8.3 and $9.0 \mu \mathrm{V}$, respectively) were regarded as zero as well, meaning that both ECU and FCU RMS values for the grip-type holder in the longitudinal directions were zero. This notion supported the development

Table 3 One-way ANOVA and multiple comparisons of RMS values of the pen-type and needle-type holders

\begin{tabular}{|c|c|c|c|c|c|c|}
\hline & \multicolumn{3}{|c|}{ Pen type } & \multicolumn{3}{|c|}{ Grip type } \\
\hline & ECU & $\mathrm{FCU}$ & Sum & ECU & FCU & Sum \\
\hline One-way ANOVA & $\mathrm{a}$ & $\mathrm{a}$ & $\mathrm{a}$ & $\mathrm{a}$ & NS & $\mathrm{a}$ \\
\hline \multicolumn{7}{|l|}{ Multiple comparison } \\
\hline $0,+30$ degrees & NS & NS & NS & a & $\mathrm{N} / \mathrm{A}$ & $a$ \\
\hline $0,-30$ degrees & $\mathrm{a}$ & NS & $\mathrm{a}$ & NS & $\mathrm{N} / \mathrm{A}$ & NS \\
\hline 0-, 90-degree antegrade & $\mathrm{a}$ & $\mathrm{a}$ & a & $\mathrm{a}$ & $\mathrm{N} / \mathrm{A}$ & $\mathrm{a}$ \\
\hline 0-, 90-degree retrograde & a & NS & a & a & $\mathrm{N} / \mathrm{A}$ & a \\
\hline$+30^{\circ},-30^{\circ}$ & a & NS & a & a & $\mathrm{N} / \mathrm{A}$ & a \\
\hline+30 -, 90-degree antegrade & a & $\mathrm{a}$ & $a$ & $\mathrm{a}$ & $\mathrm{N} / \mathrm{A}$ & $\mathrm{a}$ \\
\hline +30-, 90-degree retrograde & $\mathrm{a}$ & $\mathrm{a}$ & $a$ & $a$ & $\mathrm{~N} / \mathrm{A}$ & NS \\
\hline -30, 90-degree antegrade & a & a & a & a & $\mathrm{N} / \mathrm{A}$ & $a$ \\
\hline -30-, 90-degree retrograde & $\mathrm{a}$ & $\mathrm{a}$ & $\mathrm{a}$ & $\mathrm{a}$ & $\mathrm{N} / \mathrm{A}$ & $\mathrm{a}$ \\
\hline 90-degree antegrade, 90-degree retrograde & a & a & a & NS & $\mathrm{N} / \mathrm{A}$ & NS \\
\hline
\end{tabular}

Abbreviations: ANOVA, analysis of variance; ECU, extensor carpi ulnaris muscle; FCU, flexor carpi ulnaris muscle; N/A, not applicable; NS, not significant; RMS, root mean square.

${ }^{\mathrm{a}} \mathrm{p}<0.05$. 
concept of the grip-type holder to minimize forearm muscle movement by utilizing finger twisting.

We observed no significant difference in the relationship between the subjects' years of microsurgery experience and whether the summed RMS values were reduced by the use of the grip-type holder. However, the subjects with at least 3 years of experience tended to benefit more from its use. This indicated that the grip-type holder could have been effective in reducing the risk of coarse motion and hand shaking in experienced microsurgeons. If the grip-type holder had reduced the forearm movement of the inexperienced microsurgeons as well, using the device might have lowered the barrier for inexperienced clinicians to enter microsurgery, but unfortunately this was not the case. Since they were likely not accustomed to anastomosis under microscopic visualization, it was probable that they applied unintended force during needle advancement and thus the summed RMS values did not differ significantly between the pen-type and grip-type holders.

There is no consensus to date on the best needle holder design, with each surgeon preferring his or her own particular instrument. ${ }^{10}$ Several newly developed microsurgical needle holders have been reported, ${ }^{10-12}$ although the advantages of many devices were subjectively assessed by the authors. One such author even conceded that the usefulness of their needle holder could not be easily measured quantitatively. ${ }^{11}$ Therefore, we consider a strength of this study to be the use of objective evaluation on the merits of the newly developed needle holder.

It was unexpected that for both needle holders, needle advancement in the longitudinal directions (90-degree antegrade and 90-degree retrograde) required less forearm muscle movement than in the horizontal directions $(0,+30$, and -30 degrees) with only one exception. This finding indicated that surgeons might reduce forearm muscle movements for more stable microsurgical suturing by choosing longitudinal settings if possible.

\section{Limitations}

There are several limitations to this study. First, there is no established method to directly assess coarse motion and hand shaking during needle advancement under the microscope. Therefore, this study indirectly assessed the stability of the microsurgical needle holder tip by sEMG measurements of the ECU and FCU under the hypothesis that a decrease in forearm muscle movement would reduce coarse motion and hand shaking. Second, as this study was conducted in a laboratory setting, further research is needed on the clinical use of the grip-type microsurgical needle holder. In particular, the grip-type holder may not be suitable for operations in deep fields, such as for hepatic artery reconstruction; even if it is possible to lengthen the shaft, the direction of needle advancement could be restricted due to the instrument's single-axis design. Lastly, this study only included right-handed subjects. However, the results will also likely apply to left-handed surgeons because the shaft of the grip-type holder can rotate in a 180-degree range symmetrically from 90-degree backward to 90-degree forward.

\section{Conclusion}

Objective testing with sEMG confirmed that the newly developed grip-type microsurgical needle holder could significantly reduce forearm muscle movement as compared with a conventional pen-type needle holder. Needle advancement by finger twisting with the intrinsic muscles of hand is considered to enable this mechanism. The grip-type holder is presumably better suited for delicate microsurgical suturing, such as lymphaticovenular anastomosis or finger replantation, since the decreased forearm movement may reduce the risk of coarse motion and hand shaking.

Note

This study was presented in part at the 44th Annual Meeting of the Japanese Society of Reconstructive Microsurgery in Miyazaki, Japan, on December 7, 2017.

\section{Funding}

The authors report a grant from Nomura Medical Device Corp. during the conduct of the study.

Conflict of Interest

None declared.

\section{References}

1 Castroviejo R. Improved needle holders. Trans Am Acad Ophthalmol Otolaryngol 1952;56(06):929-930

2 Jacobson JH II. Microsurgery. Curr Probl Surg 1971;8:3-56

3 Acland RD. Instrumentation for microsurgery. Orthop Clin North Am 1977;8(02):281-294

4 Gropper PT, Kester DA, McGraw RW. Introduction to microsurgery. Clin Obstet Gynecol 1980;23(04):1145-1150

5 Ohata E, Matsuo K, Ban R, Shiba M, Yasunaga Y. Electromyographic comparison of forearm muscle movements for fine skin suturing between an enlarged pen needle holder and a Webster needle holder. Eplasty 2013;13:e22

6 De Luca CJ. The use of surface electromyography in biomechanics. J Appl Biomech 1997;13:135-163

7 Brand PW, Beach RB, Thompson DE. Relative tension and potential excursion of muscles in the forearm and hand. J Hand Surg Am 1981;6(03):209-219

8 Wang K, McGlinn EP, Chung KC. A biomechanical and evolutionary perspective on the function of the lumbrical muscle. J Hand Surg Am 2014;39(01):149-155

9 Long C II, Conrad PW, Hall EA, Furler SL. Intrinsic-extrinsic muscle control of the hand in power grip and precision handling. An electromyographic study. J Bone Joint Surg Am 1970;52(05): 853-867

10 Korula P. A modified needle holder for microsurgery. Br J Plast Surg 1990;43(04):499-501

11 Menovsky T, De Ridder D. A new flexible and $360^{\circ}$ rotating shaft needle-holder for microneurosurgery: technical note. Minim Invasive Neurosurg 2011;54(5-6):274-275

12 Matsumura N. Novel microsurgical precision grip and springhandled instrument with a variable stabilizer. Microsurgery 2011;31(07):586-588 